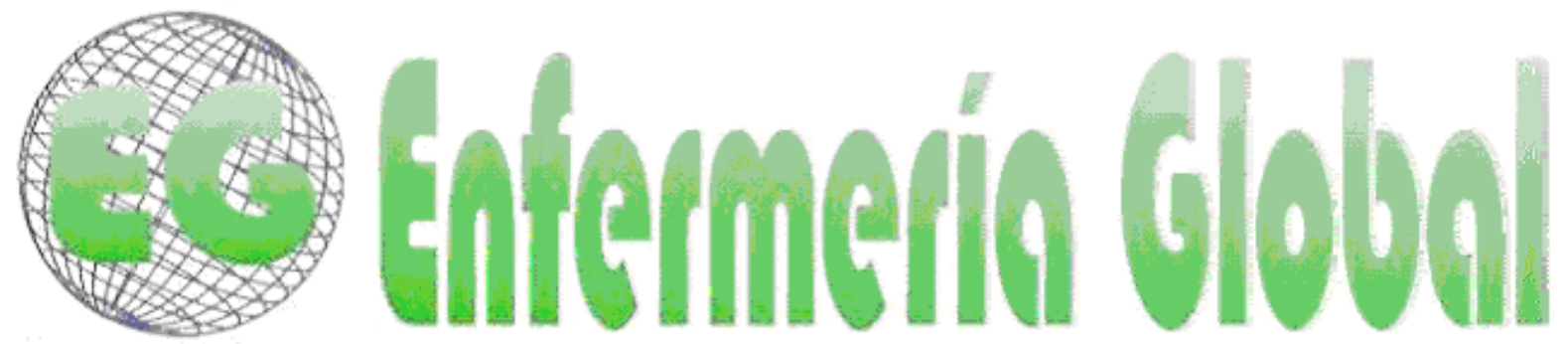

\title{
Capacidad de autocuidado de los pacientes en diálisis peritoneal: un estudio piloto en Bogotá
}

Self-care capability of the patients in peritoneal dialysis: a pilot study in Bogotá

\section{*Carrillo Algarra, Ana Julia **Díaz, Flor Janeth}

*Enfermera. Magíster en Administración Salud. Profesora Títular Facultad de Enfermería.

Coordinadora Grupo de Investigación "Perspectivas del cuidado". Investigadora principal de la investigación: "Autocuidado en pacientes en diálisis peritoneal en Unidades renales de Bogotá" . E-mail: ajcarrillo@fucsalud.edu.co ** Enfermera. Especialista en Enfermería Nefrológica y Urológica. Fundación Universitaria de Ciencias de la Salud.Facultad de Enfermería. Bogotá. Colombia.

(Este artículo presenta resultados parciales del proyecto de investigación No. 70-3278-7

“ Autocuidado en pacientes en diálisis peritoneal en Unidades renales de Bogotá" .

Financiado por la Fundación Universitaria de Ciencias de la Salud.)

Palabras clave: actividades de autocuidado; diálisis peritoneal; Dorotea Orem; insuficiencia renal crónica

Keywords: self-care activities; peritoneal dialysis; Dorothea Orem; chronic renal failure

\section{RESUMEN}

Objetivo: Medir la capacidad de autocuidado y su relación con las características sociodemográficas y clínicas en un grupo de pacientes del programa de diálisis peritoneal de acuerdo con su capacidad de autocuidado.

Métodos: Estudio descriptivo prospectivo de corte transversal. Se aplicaron tres instrumentos de recolección de datos: formulario de características sociodemográficas y clínicas, test de Morisky-Green y la escala Appraisal of Self-care Agency Scale (ASA) a 87 pacientes, hombres y mujeres, mayores de 18 años en tratamiento de diálisis peritoneal, con un mes o más de permanencia en el programa. Se realizó un análisis descriptivo que incluyó frecuencias absolutas y relativas, medidas de tendencia central y de dispersión para: el test de Morisky-Green, las variables sociodemográficas y clínicas, Se hizo análisis de correspondencias múltiples, tomando como variable ilustrativa la capacidad de autocuidado, y como activas: las variables sociodemográficas y clínicas.

Resultados: El 60\% de los sujetos reportaron suficiente capacidad de autocuidado y el $40 \%$ deficiente capacidad de autocuidado, además se determinaron las actividades de cuidado de sí mismo, que los sujetos de estudio realizan con mayor frecuencia. 


\title{
ABSTRACT
}

Objective: To measure the self-care capability and its relation with the socio-demographic and clinical characteristics in a group of patients from the peritoneal dialysis program according to their self-care capability.

\begin{abstract}
Methodology: A prospective, descriptive study of a cross-sectional nature was done. Three data recollection instruments were applied: A socio-demographic and clinical characteristics form, the Morisky-Green test and the Appraisal of Self-care Agency Scale (ASA) were used with 87 patients, male and female alike. They were all over 18 years old and they were going through a peritoneal dialysis treatment having been over a month in the program. A descriptive analysis was done which included absolute and relative frequencies, central tendency and dispersion measures for the MoriskyGreen test and for the socio-demographic and clinical tendencies. A multiple correspondence analysis was also done, using as the illustrative variable the self-care capability and as the control differences the following: The socio-demographic and clinical variables.
\end{abstract}

Results: $60 \%$ of subjects reported sufficient self-care capability and the remaining $40 \%$ reported an insufficient self-care capability. In addition, the self-care activities that the subjects perform the most were defined.

\section{INTRODUCCIÓN}

"El autocuidado es una conducta que existe en situaciones concretas de la vida, dirigida por las personas sobre sí mismas, hacia los demás o hacia el entorno, para regular los factores que afectan su propio desarrollo y funcionamiento en beneficio de su vida, salud o bienestar" (1)

Cuidar de sí mismo es muy importante cuando se refiere a pacientes con Insuficiencia Renal Crónica (IRC), entidad que ha sido considerada en el Sistema General de Seguridad Social en Salud (SGSSS) colombiano como una enfermedad de alto costo por generar un fuerte impacto económico y causar un efecto nocivo para la calidad de vida del paciente y su familia, incluidas las repercusiones laborales; se estima que su manejo ha comprometido en los últimos años aproximadamente el $2 \%$ del gasto en salud del país y el $4 \%$ del gasto en seguridad social en salud. Según datos del Ministerio de la Protección social, en la cuenta de alto costo para el 2011, se reportaron 640.492 personas afectadas por esta enfermedad, con una tasa de mortalidad del $10.63 \%$ por 100.000 afiliados ${ }^{(2)}$

Teniendo en cuenta la magnitud del problema, las actividades de autocuidado son importantes por la influencia que ejercen en el control de los principales factores de riesgo que originan complicaciones en el paciente que se encuentra en diálisis peritoneal; si las personas adquieren educación oportuna, adecuada e individualizada, integrando aspectos físicos, emocionales y sociales, se espera que se hagan responsables del cuidado de su propia salud.

El conocimiento acerca de cómo cuidarse y las habilidades para hacerlo, aumentan la capacidad de las personas y familias para evaluar su estado de salud y determinar la necesidad de buscar atención profesional, así lo concluyó Sánchez en un estudio realizado con pacientes con cardiopatía isquémica cuaándo escribe, "El apoyo educativo que proporciona el personal de enfermería al paciente influye positivamente en su capacidad de autocuidado" (3) porque permite tener conciencia, atender los efectos de las enfermedades y cumplir las prescripciones médicas, diagnósticas y las medidas de rehabilitación específicas.

Es necesario recordar que la percepción de necesidad de cuidado es única para cada persona, al igual que las acciones y el modo en que cada uno las realiza para 
cuidar de si mismo porque están determinadas por los hábitos y costumbres, es decir, por las condiciones sociales ${ }^{(4,5,6)}$ aspecto relevante para la persona con IRC quien debe modificar su vida para adaptarse a la enfermedad. Por lo anterior, el propósito del estudio piloto fue medir la capacidad de autocuidado y su relación con las características sociodemográficas y clínicas en un grupo de pacientes del programa de diálisis peritoneal en dos instituciones prestadoras de servicios de salud de Bogotá, Colombia.

Los resultados son útiles para orientar a los profesionales de salud, en el diseño de planes de cuidado y educación específicos, que le permitan a las personas en diálisis peritoneal compensar el déficit de autocuidado, igualmente, se espera que influyan en la calidad de vida de los pacientes y sus cuidadores no profesionales con la disminución de complicaciones prevenibles. Además, beneficiará en forma indirecta la economía de los países, con la disminución de costos generados por complicaciones evitables con actividades de autocuidado.

\section{MATERIALES Y MÉTODOS}

Estudio descriptivo prospectivo de corte transversal en el cual se entrevistaron 87 pacientes hombres y mujeres, mayores de 18 años, en tratamiento de diálisis peritoneal ordenado por médico nefrólogo, con un mes o más de permanencia en el programa de diálisis peritoneal en dos unidades renales de Bogotá, Colombia, durante el periodo marzo-septiembre de 2011. Se excluyeron las personas con alteraciones previas o adquiridas durante el tratamiento que les impedía cuidarse y los pacientes que no aceptaron participar en el estudio. El muestreo utilizado fue no probabilístico por conveniencia.

Previo consentimiento informado y garantía de anonimato, enfermeras que estaban cursando el último semestre de la especialización en Enfermería Nefrológica y Urológica aplicaron tres instrumentos:

- Un cuestionario para medir las características sociodemográficas y variables clínicas de los pacientes.

- El "Test de Morisky Green" para determinar la adherencia al tratamiento.

- La escala Appraisal of Self-care Agency Scale (ASA) propuesta por Isemberg en 1983, ${ }^{(7)}$ traducida al español como "Escala de Valoración de Agencia de Autocuidado (ASA)", que es un instrumento de 24 ítems, tipo escala de Likert, modificado para Colombia por Edilma de Reales, con autorización de la autora, y validado por Manrique y Velandia, para la población bogotana ${ }^{(8)}$, la puntuación 1 corresponde a nunca, 2 a casi nunca, 3 a casi siempre y 4 a siempre, por tanto, el sujeto con máxima capacidad de autocuidado tendrá un puntaje de 96 y el mínimo de 24 . Los datos se analizaron utilizando frecuencias absolutas y relativas, medidas de tendencia central y de dispersión para las variables sociodemográficas y clínicas, dependiendo de su naturaleza. La clasificación de la capacidad de autocuidado se realizó con base en lo descrito por Rivera y Díaz, quienes plantean dos categorías según corte generado por el promedio así:

-Si el valor total para cada persona es igual o superior al promedio, se clasifica como suficiente capacidad de autocuidado.

-Si el valor total para cada persona es inferior al promedio, se clasifica como deficiente capacidad de autocuidado. ${ }^{(9)}$

Se hizo análisis de correspondencias múltiples, tomando como variable ilustrativa la capacidad de autocuidado, y como activas: las variables sociodemográficas y 
clínicas ${ }^{(10)}$ con el fin de determinar los ítems que requieren mayor atención en el momento de planear programas de educación o realizar planes de cuidado, se construyó una grafica de perfiles que permitió identificarlos fácilmente. Para el análisis estadístico de la información se utilizó Stata $12 \AA$ y Spad 7.3®.

Para la ejecución del estudio, se tuvo en cuenta la Resolución 8430 de 1993 del Ministerio de Salud de la República de Colombia ${ }^{(11)}$. El proyecto fue aprobado por el Comité de Investigación de la Facultad de Enfermería de la Fundación Universitaria de Ciencias de la Salud. Una vez firmado el consentimiento informado por cada uno de los sujetos de estudio, la información fue recolectada por estudiantes de tercer semestre de la especialización de Enfermería Nefrológica y Urológica, quienes inmediatamente la obtuvieron elaboraron con cada persona el plan de cuidado enfocado a compensar el déficit en la capacidad de autocuidado.

\section{RESULTADOS}

\section{Características sociodemográficas de la población de estudio.}

En la revisión de los registros institucionales se encontraron 94 pacientes que cumplían con los criterios de inclusión, 4 de ellos no asistieron al control y 3 no aceptaron participar, por lo tanto los instrumentos se aplicaron a 87 pacientes: 42 de la unidad renal 1 y 45 de la unidad 2.

La edad promedio de los sujetos de estudio fue de 54,7 años (DE: 14.4 ); el $51,7 \%$ de la población fue de sexo masculino y 48 participantes estaban casados; predominan los estratos socioeconómicos 2 y 3 . La mayoría de los participantes realizó la primaria, 24 persona estaban desempleadas y 24 dedicadas a oficios varios. (Tabla I).

[Tabla I. Características socio demográficas de la población de estudio]

\begin{tabular}{|lrl|}
\hline Estado civil & $\mathrm{n}(87)$ & $(\%)$ \\
Soltero & 13 & 14.94 \\
Casado & 48 & 55.17 \\
viudo & 10 & 11.49 \\
unión libre & 12 & 13.79 \\
separado & 4 & 4.6 \\
Nivel socioeconómico & & \\
Estrato 1 & 12 & 13.79 \\
Estrato2 & 40 & 45.98 \\
Estrato 3 & 32 & 36.78 \\
Estrato 4 & 3 & 3.45 \\
Nivel educativo & & \\
Sin estudios & 2 & 2.3 \\
Primaria & 37 & 42.53 \\
Bachillerato & 34 & 39.08 \\
Técnico & 4 & 4.6
\end{tabular}




\begin{tabular}{|lrr|} 
Universitario & 10 & 11.49 \\
Ocupación & & \\
Empleado & 10 & 11.49 \\
Desempleado & 24 & 27.59 \\
Pensionado & 18 & 20.69 \\
Trabajador independiente & 11 & 12.64 \\
Oficios varios & 24 & 27.59 \\
\hline
\end{tabular}

Fuente: La Investigadoras, 2011.

\section{Características clínicas de la población de estudio.}

La media del tiempo de permanencia en diálisis fue de 36 meses y el antecedente familiar reportado con mayor frecuencia fue la hipertensión arterial. Según la etiología de la IRC, la mayoría se consideró de origen diabético. Los datos reportaron, que el $57 \%$ de las personas hacen ejercicio, el $62 \%$ tiene compromiso con el tratamiento farmacológico y el $78 \%$ sigue la dieta indicada. (Tabla II).

[Tabla II. Características clínicas de la población de estudio]

\begin{tabular}{|lcc|}
\hline & $\mathrm{n}(87)$ & $(\%)$ \\
Percepción del estado de salud & & \\
Malo & 4 & 4.6 \\
Regular & 35 & 40.2 \\
Bueno & 47 & 54.0 \\
Muy bueno & 1 & 1.2 \\
Antecedentes familiares & & \\
Ninguno & 18 & 20.69 \\
Diabetes & 20 & 22.99 \\
Hipertensión Arterial & 32 & 36.78 \\
Diabetes + Hipertensión Arterial & 17 & 19.54 \\
Antecedentes personales & & \\
Ninguno & 4 & 4.65 \\
Diabetes & 9 & 10.47 \\
Diabetes + Hipertensión Arterial & 24 & 27.91 \\
Hipertensión Arterial & 43 & 50 \\
Hipertensión Arterial + dislipidemia & 4 & 4.65 \\
Hipertensión Arterial + Síndrome coronario & 1 & 1.16 \\
Síndrome coronario & 1 & 1.16 \\
Etiología de la IRC & & \\
Diabetes & 31 & 35.63 \\
Diabetes + Vascular & 19 & 1.15 \\
Glomerular & 21.84 \\
\hline
\end{tabular}




\begin{tabular}{lcc|} 
Glomerular + Quística & 1 & 1.15 \\
Vascular & 19 & 21.84 \\
Quística & 1 & 1.15 \\
Otras & 15 & 17.24 \\
Ejercicio & 50 & 57.47 \\
Dieta adecuada & 68 & 78.16 \\
Adherencia al tratamiento farmacológico & 54 & 62.07 \\
$\begin{array}{l}\text { Tiempo de diálisis (meses) mediana } \\
\text { (RIQ†) }\end{array}$ & 36 & $(18-60)$ \\
\hline
\end{tabular}

Fuente: Las Investigadoras, 2011.

\section{Capacidad de autocuidado en la población de estudio}

Se hizo análisis de correspondencias múltiples, tomando como variable ilustrativa la capacidad de autocuidado, y como activas: las variables sociodemográficas y clínicas. El resultado promedio de la escala ASA fue de 76.4 puntos (DE: 5.5). Tomando este dato como punto de corte de acuerdo con Rivera y Díaz ${ }^{(9)}$, el $60 \%$ de los sujetos reportan suficiente capacidad de autocuidado y el $40 \%$ deficiente. El análisis por ítem mostró que 36 personas contestaron que "Nunca" pueden buscar mejores forma de cuidar su salud que las que tienen ahora; la valoración "Casi nunca" fue asignada por 25 personas al ítem 11 "Pienso en hacer ejercicio y descansar un poco durante el día pero no llego a hacerlo." (Figura 1)

[Figura No. 1. Perfil de la capacidad de autocuidado según la Escala de ASA de la población de estudio]

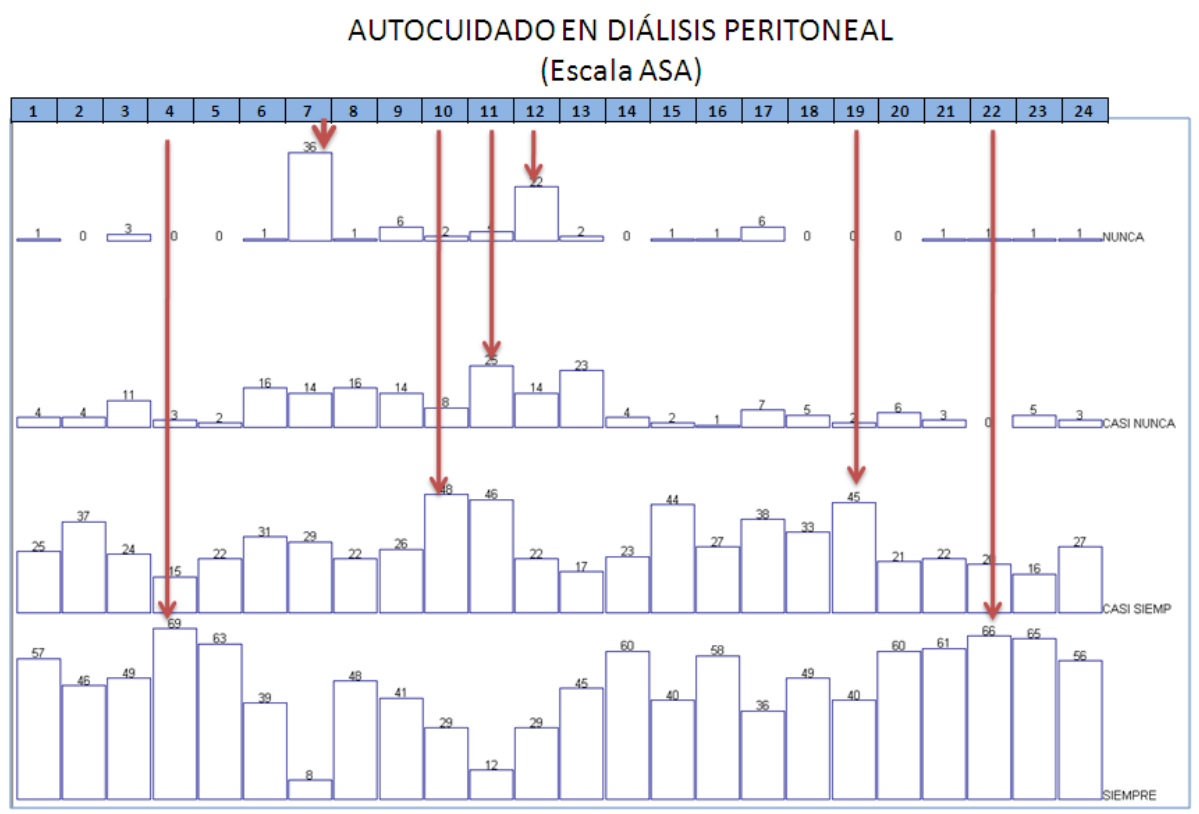

Fuente. Las investigadoras, 2011

La respuesta "Casi siempre" fue asignada por 48 personas al ítem "Cuando hay situaciones que me afectan yo las manejo de manera que pueda mantener mi forma 
de ser" y 69 personas manifestaron que "Siempre" pueden hacer lo necesario para mantener limpio el ambiente donde viven. (Figura1)

\section{Caracterización de las variables clínicas y sociodemográficas con relación a la} capacidad de autocuidado

Los resultados reportados por la escala ASA con relación a las variables sociodemográficas y clínicas muestran que los participantes que clasifican en autocuidado deficiente se caracterizan por ser personas que se dedican a los oficios varios o empleados con un nivel educativo de bachillerato; son separados y con menor frecuencia solteros, tienen antecedentes personales de hipertensión arterial y dislipidemia, manifiestan no cumplir el tratamiento farmacológico y consideran que su estado de salud es bueno. (Figura 2)

[Figura 2. Caracterización sociodemográficas y clínica de los pacientes en diálisis peritoneal con respecto al ASA suficiente o deficiente]

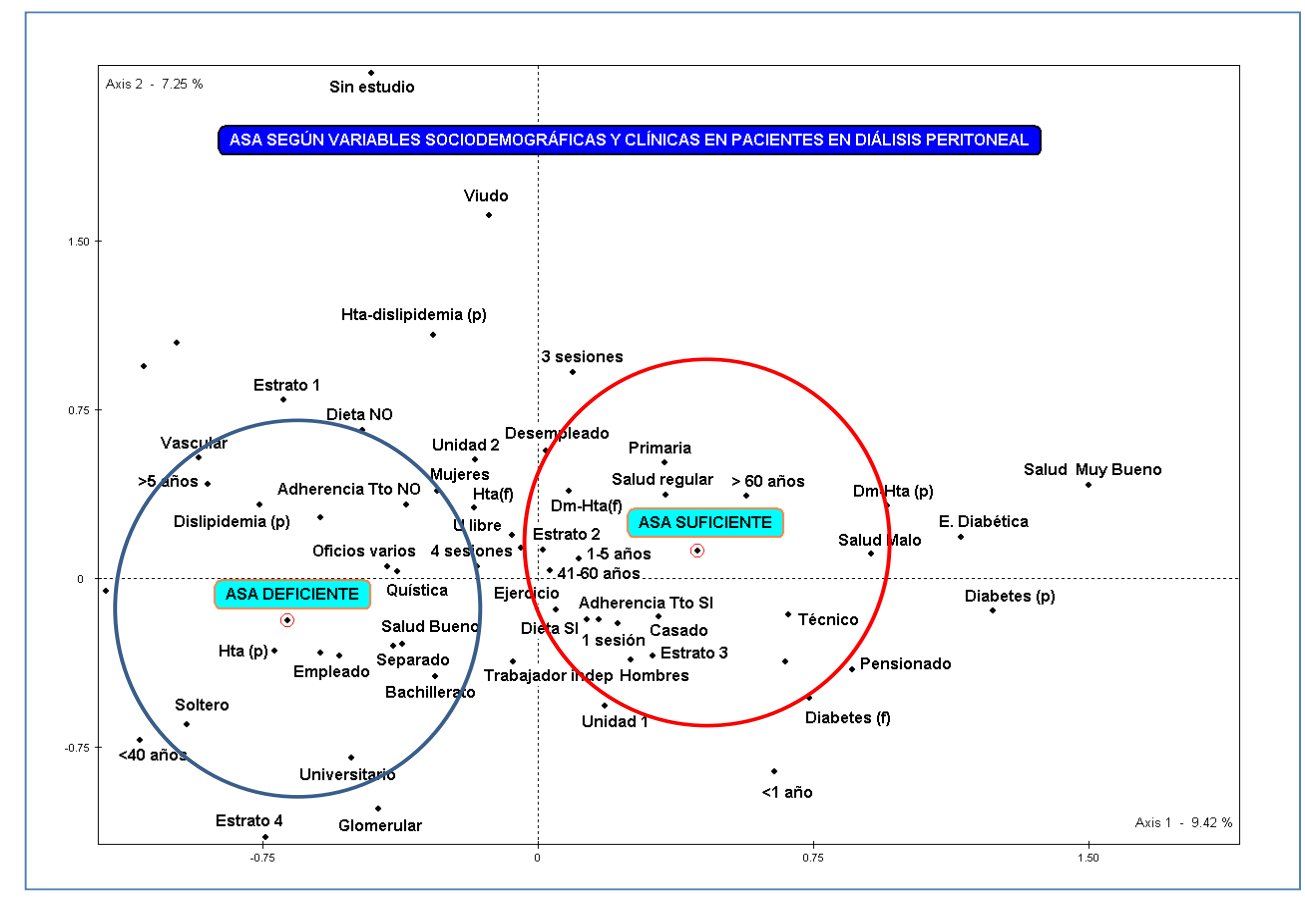

Variable Ilustrativa: Capacidad de autocuidado.

Variables activas: Edad, nivel socioeconómico, nivel educativo, ocupación, precepción del estado de salud, antecedentes familiares, antecedentes personales, etiología de la IRC, ejercicio, dieta adecuada, adherencia al tratamiento farmacológico, tiempo de diálisis en meses.

Por otro lado las personas que clasificaron en la categoría capacidad de autocuidado suficiente, se caracterizan por ser mayores de 41 años, con un nivel educativo de primaria o técnica, pertenecer a los estratos 2 y 3 , se encuentran casados, reportan cumplimiento del tratamiento farmacológico y la dieta establecida, hacen ejercicio, manifiestan como antecedente familiar y personal diabetes e hipertensión arterial, y perciben su estado de salud como regular o malo. (Figura 2) 


\section{DISCUSION}

La edad promedio de los participantes en el estudio fue de 54,7 años con una (DE: 14.4) años, resultado coherente con las estadísticas nacionales que reportan la mayor frecuencia de IRC en pacientes con más de 50 años ${ }^{(2)}$. El $51,7 \%$ de la población es de sexo masculino y el mayor porcentaje de los participantes al momento del estudio estaban casados, lo cual es esperable teniendo en cuenta la edad de los participantes. Con respecto al nivel socioeconómico, el mayor porcentaje se encuentra en los estratos socioeconómicos 2 y 3 , ${ }^{(12)}$ que concuerda con lo encontrado en otros estudios de personas en condición de cronicidad como el de Manrique ${ }^{(8)}$.

En el momento del estudio, el $42.53 \%$ de las personas habían finalizado la educación primaria y el $39.08 \%$ el bachillerato, resultado coherente con los estratos a los cuales pertenece la mayor parte de la población; el mayor porcentaje de las personas del estudio se encontraba distribuido entre desempleados y dedicados a oficios varios, lo cual se relaciona con la modificación en los roles que genera la condición de persona con IRC descrita por Gutiérrez ${ }^{(5)}$ a pesar de lo expresado por Alarcón cuando afirma que dentro de los pacientes en terapia de sustitución renal los que más conservan sus trabajos son los que se encuentran en diálisis peritoneal ${ }^{(13)}$.

Con respecto a las variables clínicas, llama la atención que a pesar de su condición de personas en situación de cronicidad, con un promedio de permanencia en diálisis de 36 meses, el $57 \%$ de los sujetos del estudio perciben su estado de salud como bueno, aspecto que merece especial atención frente a la primera fase de elaboración del duelo que es la negación, en la cual como lo plantea Alarcón, la persona se crea una fantasía de que la realidad no está ocurriendo, muchas veces se suprime de la conciencia la situación dolorosa, o se niega la seriedad de su impacto sobre la vida, incluso han encontrado casos de personas que ignoran sus síntomas por varios años, lo que puede ser causa de no seguir los tratamientos ni cuidados recomendados ${ }^{(13)}$

La hipertensión arterial fue reportada por el $36.78 \%$ de las personas como antecedente familiar seguida muy de cerca de la diabetes, resultado concordante con la literatura ${ }^{(2)}$, con los antecedentes personales y lo reportado en la historia clínica sobre la etiología de la enfermedad renal crónica en el grupo de estudio, que en la mayoría se consideró de origen diabético, seguida del vascular y el quístico. Los datos reportaron que el $57 \%$ de las personas hace ejercicio, el $62 \%$ tiene adherencia al tratamiento farmacológico y el $78 \%$ sigue la dieta, porcentajes muy cercanos a la capacidad de autocuidado y al reporte del estudio realizado por Alarcón. Estos resultados sugieren que los pacientes con mayor comprensión de las restricciones alimentarias y de las razones del tratamiento logran mayor adherencia al tratamiento e incrementan la capacidad para cuidar de sí mismos ${ }^{(13,14)}$.

Con relación a la capacidad de autocuidado, la media aritmética reportada por la escala ASA, fue de 76.4 puntos (DE: 5.5 ), cuando se relaciona este dato con los hallazgos del test de Morisky-Green y los obtenidos por Rivera y Díaz, en pacientes con Hipertensión arterial, se ratifica la capacidad de la escala ASA para medir las acciones de autocuidado que tienen las personas que sufren una enfermedad crónica $^{(9)}$

Según los perfiles obtenidos con base en los resultados de la escala ASA, el ítem que con mayor frecuencia reportan que "Nunca" realizan 36 los pacientes es: "Yo puedo buscar mejores forma de cuidar mi salud que las que tengo ahora", lo cual sugiere que hay un grupo de personas con IRC que: aceptan la enfermedad, saben que deben realizar acciones tendientes a modificar sus acciones de cuidado, pero no lo hacen; lo cual indica que es necesario revisar los procesos educativos desarrollados 
por el personal de la salud, teniendo en cuenta la afirmación de Alarcón ${ }^{(13)}$ quién plantea que las fallas en la educación por parte del personal de salud, sobre la importancia del cumplimiento en la toma de medicamentos y la dieta han sido asociadas con menor compromiso con el tratamiento. Además 22 personas manifestaron que "nunca" pueden recurrir a sus amigos de siempre cuando necesitan ayuda, lo cual puede ser resultado de los cambios de vida que tiene que realizar el paciente que se encuentra en diálisis peritoneal, que incluye cambio en sus actividades sociales. La respuesta "casi nunca" fue asignada por 25 personas al ítem "pienso en hacer ejercicio y descansar un poco durante el día pero no llego a hacerlo", éste ítem es inverso lo cual quiere decir que las 25 personas sí hacen ejercicio y descansan durante el día, lo que sugiere la necesidad de lograr mayor eficacia en el apoyo educativo; 23 participantes en el estudio manifiestan que "casi nunca" pueden dormir lo suficiente como para sentirse descansados, genera en el personal de salud la necesidad de enseñar al paciente estrategias de relajación y técnicas para conciliar el sueño con el fin de compensar este déficit.

La respuesta "casi siempre" fue asignada por 48 personas al ítem "Cuando hay situaciones que me afectan yo las manejo de manera que pueda mantener mi forma de ser", respuesta que coincide con lo que dice Alarcón, quien refiere que dado que la diálisis peritoneal es una actividad terapéutica que desarrollan los pacientes por sí mismos, se requiere que ellos tengan un alto nivel de autonomía ${ }^{(13)}$. Igualmente 46 personas contestaron que casi siempre "piensan en hacer ejercicio y descansar un poco pero no llegan a hacerlo," es necesario recordar que este ítem es inverso ${ }^{(8)}$ y por lo tanto muestra que las personas que lo contestaron como casi siempre no hacen ejercicio y no descansan lo suficiente, aspectos que permiten identificar la necesidad de incrementar el apoyo educativo proporcionado por el personal de enfermería, teniendo en cuenta lo descrito por Kutner y cols. quienes observaron que el nivel de actividad física en los pacientes en tratamientos sustitutivos puede predecir el nivel de calidad de vida de los mismos ${ }^{(15)}$. El ítem "soy capaz de evaluar que tanto me sirve lo que hago para mantenerme con salud" fue respondido como casi siempre por 45 sujetos, lo cual sugiere que tienen un grado de autonomía que favorece el buen desarrollo de la diálisis peritoneal.

Como "siempre se ejecuta", 69 personas reportaron el ítem "Yo puedo hacer lo necesario para mantener limpio el ambiente donde vivo", resultado que sugiere el efecto positivo de la educación proporcionada por el personal de salud, 66 personas respondieron que siempre ejecutan el ítem 22 "si yo no puedo cuidarme puedo buscar ayuda" reafirmando la importancia de las redes de apoyo y 65 personas manifiestan; siempre "Puedo sacar tiempo para mí", resultados que ratifican la importancia del apoyo educativo, que permite a las personas identificar sus necesidades y las mejores formas de satisfacerlas, y coherentes con lo reportado por Carballo cuando infiere que la técnica didáctica utilizada por la enfermera ofrece buenos resultados ${ }^{(16)}$

Los resultados reportados por la escala ASA en relación con las variables sociodemográficas y clínicas, permiten identificar que las personas con clasificación deficiente se caracterizan por ser personas que se dedican a los oficios varios 0 trabajan como empleados, lo cual sugiere que los programas educativos deben ser de fácil comprensión para que sean eficaces en la enseñanza de acciones de autocuidado y puedan desarrollar el componente de poder descrito por Orem cuyos elementos son la motivación, adquisición de conocimientos, habilidad para ordenar acciones de cuidado para sí mismo y capacidad de integración del autocuidado en su vida familiar y comunitaria ${ }^{(17)}$. Otra característica de las personas con clasificación deficiente es que son separadas y con menor frecuencia solteras, lo cual demuestra la necesidad de contar con redes de apoyo que fortalezcan el deseo de cuidarse, al 
respecto, Alarcón plantea que el percibir una red fuerte, de apoyo social, se asocia con tasas de supervivencia más altas, al parecer mediadas por un mayor compromiso con el tratamiento que depende en gran parte del paciente, pero tiene gran influencia del equipo de salud, el cual, debe determinar en cada persona que barreras existen para lograrlo, y cómo pueden ser superadas ${ }^{(13)}$.

Los antecedentes personales que reportaron con mayor frecuencia fueron hipertensión arterial y dislipidemia, enfermedades que requieren acciones de autocuidado para su control. Igualmente este grupo de participantes manifestó no adherencia al tratamiento farmacológico y consideró que su estado de salud era bueno, lo cual según Alarcón podría tener alguna relación con la etapa de negación en el proceso de elaboración del duelo o indicar alteración en la percepción de la salud $^{(13)}$

\section{CONCLUSIONES}

Las personas que clasificaron en ASA suficiente se caracterizaron por ser mayores de 41 años, con un grado educativo de primaria o técnica, pertenecían a los estratos 2 y 3, se encontraban casados, reportaban adherencia al tratamiento farmacológico, hacían ejercicio, cumplían con la dieta establecida y percibían su estado de salud como regular o malo, respuestas que sugieren el grado de conciencia que tenían de la enfermedad y la importancia de cuidarse. Estos resultados fueron coherentes con lo descrito por Alarcón quién plantea que los pacientes que tienen mayor comprensión de las restricciones alimentarias y las razones del tratamiento presentan un mayor compromiso con el tratamiento, además sugiere que la técnica educativa ofrece buenos resultados $(16,17)$

\section{REFERENCIAS}

1. Marinner A. Raile M. Modelos y Teorías de Enfermería. Elsevier-Mosby. Sexta Edición,2007. P. 271.

2. República de Colombia. Ministerio de Protección social. Cuenta de alto costo pacientes con enfermedad renal crónica en diálisis peritoneal. Bogotá, 2011.

3. Sanchez, C. Influencia del apoyo educativo de enfermeria en el autocuidado del paciente con cardiopatía isquémica. Desarrollo científico de enfermería vol. 12 № 2 , marzo de 2004

4. Organización panamericana de la salud .Organización mundial de la salud, Consejo Directivo $58^{a}$ sesión del comité regional, Washington, D.C., EUA, 25-29 de septiembre 2006 .disponible en /es.scribd.com/doc/66197150/Carta-Otawa-Salud.

5. Gutiérrez Vilaplana, Josep $\mathrm{M}^{\mathrm{a}}$ et al. Evaluación de la intervención enseñanza: grupo en la consulta de enfermedad renal crónica avanzada. Rev Soc Esp Enferm Nefrol [online]. 2007, vol.10, n.4 [citado 2012-06-26], .24-29.

6. López Ortega, J et al. Aproximación a los cuidados informales de enfermos renales crónicos en el complejo hospitalario de Jaén. Rev Soc Esp Enferm Nefrol [online]. 2007, vol.10, n.2 [citado 2012-06-26];55-64. Disponible en: $<$ http://scielo.isciii.es/scielo.php?script=sci_arttext\&pid=S1139-

7. Evers. G.C. Eisemberg, M.A. Philipsen.H. Senten M. Brouns. M.N.S. Validity Testing of the Dutch translation of the appraisal of the self-care Agency A.S.A. Scale. Int.J.Nurs Stud. 1993. Vol. 30 № 4; 331-42. 
8. Manrique F, Fernández A, Velandia A. (2009). Análisis factorial de la escala de valoración de la agencia de autocuidado. Aquichan, vol. 9, №3, diciembre de 2009; 203-235.

9. Rivera LN, Díaz, LP. Relación entre la capacidad de agencia de autocuidado y los factores de riesgo cardiovascular. Cuad. - Hosp. Clín., jul. 2007, vol.52, №.2; 30-38. ISSN 1652-6776

10. Greenacre Michael. La práctica del análisis de correspondencias. Capítulo I. Diagramas de dispersión y mapas. Edición en Español. Fundación BBVA, 2008. http://www.fbbva.es/TLFU/tlfu/esp/publicaciones/libros/fichalibro/index.jsp?codigo=300

11. Normas científicas, técnicas y administrativas para la investigación en salud. Resolución 0084304 de octubre de 1993

12. DANE. Coordinación de Proyecciones de Población y Estudios Demográficos. Indicadores Demográficos Colombia 2005. Disponible en: www.consultor salud.com/biblioteca/documentos.

13. Alarcón A. Aspectos psicosociales del paciente renal, segunda edición, 2004, Bogotà, Colombia.. 111-116.

14. Bezerra, Karina Viviani y Ferreira Santos, Jair Licio. (2008). El día a día de personas con insuficiencia renal crónica. Sao Paulo: Revista latinoamericana Enfermagen.

15. Kutner NG, Zhang R. MCCLELAN WM, COLE SA ,Phycosocial predictors of no compliance Hemoialysis and peritonealdialysis patients, Nwfphrol Dial Trasnplantation (2002) 17:93-99

16. Carballo MR, Ortega N, Lizárraga EC, Díaz J. Adherencia individual y familiar al tratamiento de diálisis peritoneal ambulatoria continua. Rev Enferm Inst Mex Seguro Social 2008; 16(1): 13-18

17. Achury DM, Sepúlveda G. Rodríguez SM, Validez de apariencia y de contenido de un instrumento para evaluar la capacidad de agencia s de autocuidado en el paciente con hipertensión arterial. Investigación en Enfermería Imagen y Desarrollo, vol 10, nùmero1, enero-junio 2008;93-111 